\title{
Power Conversion Efficiency Characterization and Optimization for Smartphones
}

\author{
Woojoo Lee Yanzhi Wang \\ Pedram \\ University of Southern California, CA, USA, *Seoul National University, Korea, \\ $\dagger\left\{\right.$ woojoole, yanzhiwa, pedram\}@usc.edu, ${ }^{*}\{$ dhshin, naehyuck\}@elpl.snu.ac.kr
}

\begin{abstract}
Modern smartphones consume significant power and can hardly provide a full day's use between charging operations even with a $2000 \mathrm{mAh}$ battery. This is in spite of many power management techniques being employed in the smart phones. This paper starts from the observation that modern smartphones waste a significant amount of the battery's stored energy during power conversion from the $3.7 \mathrm{~V}$ output of a Li-Ion battery cell to different voltage levels needed to power various modules in a smartphone (processors, memory, display, GPS, etc.) Indeed the power conversion efficiency from the battery source to point of use in the smart phone has on average of only $60-75 \%$ efficiency. The approach taken to reduce this energy waste in smartphones is to (i) profile the power consumption of each module under different operating scenarios, (ii) build an equivalent DC-DC converter model for each smartphone module and estimate its power conversion efficiency, and (iii) change the parameters of the actual converters in the smartphone to improve the equivalent power conversion efficiencies for all modules. Experimental results demonstrate that we can achieve $6 \%$ to $15 \%$ power conversion efficiency enhancement, which translates to up to $30 \%$ reduction in the power losses incurred during power conversion in smartphones.
\end{abstract}

\section{Categories and Subject Descriptors}

C.5.3 [Computer system implementation]: Microcomputers

\section{Keywords}

Portable device, Smartphone, DC-DC converter, Power tree

\section{INTRODUCTION}

Modern smartphones are typically equipped with a multi-core gigahertz processor, gigabytes of high-speed DDR SDRAM, dozens of gigabytes of flash memory, several up to 10 megapixel cameras, $1 \mathrm{M}+$ pixel high-resolution color display, high-power audio, as well as $3 \mathrm{G} / 4 \mathrm{G}$, Wi-Fi and Bluetooth wireless communication devices. Consequently, their power consumption is as high as a small-size notebook computer or a tablet. Although the smartphone battery capacity has been increased from several hundred $\mathrm{mAh}$ to over $2000 \mathrm{mAh}$ over the last few years, the actual battery life (i.e., the service time between consecutive charges) has become

Permission to make digital or hard copies of all or part of this work for personal or classroom use is granted without fee provided that copies are not made or distributed for profit or commercial advantage and that copies bear this notice and the full citation on the first page. To copy otherwise, to republish, to post on servers or to redistribute to lists, requires prior specific permission and/or a fee.

ISLPED'12, July 30-August 1, 2012, Redondo Beach, CA, USA.

Copyright 2012 ACM 978-1-4503-1249-3/12/07 ...\$10.00.

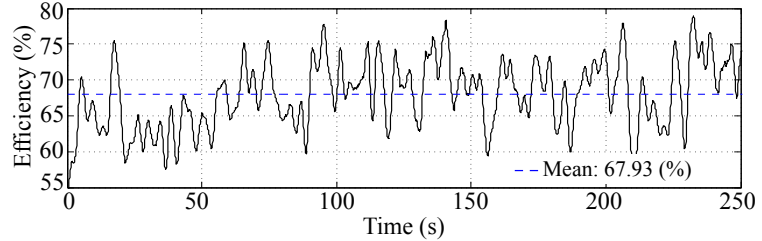

Figure 1: Measured of traces of power conversion efficiency of Qualcomm Snapdragon MDP MSM8660.

shorter due to even faster increase in the power consumption of the smartphones. An examination of the technology trends reveals that smartphone system-on-chips are migrating from dual cores to quad cores, display sizes continue to increase with some high-end smartphones having over 5" display, wireless data communication rates are increasing rapidly with a100MHz aggregated bandwidth, 4G LTE-Advanced providing almost 3.3G Git peak download rates per sector of the base station, and so on. All this will result in significantly higher power consumption in the smartphones and a smaller battery life (active users of a recent $4 \mathrm{G}$ smartphone claim less than 2-3 hours of battery life.) High power consumption of smartphones (and relatively slow rate of increase in the energy storage density of batteries) is thus a serious concern that could derail the smartphone technology development and adoption.

It is not surprising that system-level power optimization and management have been widely investigated and advanced over the last two decades. Some recent works have explicitly focused on smartphones. In particular, references $[1,2,3,4]$ have presented various power dissipation models for the smartphones. Dynamic voltage (and frequency) scaling techniques have also been used to reduce the power consumption of various smartphone modules, including the CPU [5] and the display [6]. LCD backlight scaling has also been widely investigated [7].

Power optimization and management works for smartphones have targeted power savings in embedded processors, memory, display, and so on. None has addressed the issue of power conversion efficiency in the smartphones. This is an important problem that has gone unnoticed. More precisely, modern smartphones are equipped with many modules, each requiring its own supply voltage level which is typically different from those of other modules in the system. The smartphone is powered by a secondary (rechargeable) LiIon battery comprised of a single battery cell providing an initial output voltage level of $4.2 \mathrm{~V}$ for a fully charged cell and as low as $3.0 \mathrm{~V}$ for a nearly discharged battery (this yields the familiar nominal average output voltage level of 3.6 or $3.7 \mathrm{~V}$ at $0.5 \mathrm{C}$ discharge rate.) This cell output voltage must be converted and regulated to different pre-determined voltage levels and distributed to various modules in the smartphone.

We have performed extensive measurement of power conversion efficiency for Qualcomm's smartphone platform, MDP MSM8660, as shown in Figure 1. Surprisingly, the power conversion efficiency of MDP MSM8660 ranges just from below 60\% to slightly over $75 \%$. Improving the power conversion efficiency can achieve significantly longer battery life. This paper thus focuses on power con- 
version efficiency in smartphones and introduces power conversion efficiency characterization and optimization procedures.

Modern DC-DC converters exhibit very high peak conversion efficiency, but their conversion efficiency can drop dramatically due to the operating conditions, i.e., their output current level [8]. In other words, a low overall conversion efficiency of DC-DC converters is mainly due to mismatch between the power converter characteristics and the load demands.

In this paper we propose a general equivalent $D C$ - $D C$ converter model to model different types of converters (such as a buck DCDC converter, a boost DC-DC converter, low-dropout (LDO) converter) and their series combinations in a power delivery path from the battery cell to the load device. In particular, we use the DC-DC converter model from [8] and identify coefficients of the equivalent power converter models with the aid of the application profiling tool named Trepn ${ }^{\mathrm{TM}}$.

We propose device grouping to enhance the accuracy of linear regression used for power efficiency characterization of the equivalent DC-DC converter. We then verify the accuracy of the power conversion efficiency characterization with real measurements. The results point to the fact that power conversion efficiency of the smartphone platform is quite low. Next, we model and verify the current demand distribution for each module in the smartphone platform and derive its expected value. Finally, we adjust the DCDC converter parameters to ensure that the power converters operate at the most efficient points. Experimental results demonstrate that we can achieve $6 \%$ to $15 \%$ power conversion efficiency enhancement, which translates to up to $30 \%$ reduction in the power losses incurred during power conversion in smartphones.

Notice that the proposed flow can be exploited to do optimization in presently available commercial smartphone platforms, which do not have current sensors to report the component current demands under different applications and usage scenarios. This is possible because we make use of activity profiling-based power estimation $[1,2,3,4]$.

\section{POWER CONVERSION LOSS MODELS}

Given that the smartphones integrate hardware modules that have their own supply power lines and require their own voltages, it is necessary to generate different voltages for various modules from a single battery that powers the smartphone. A power conversion tree, with the root of the tree being the battery cell, the leaf nodes being the modules, and the internal nodes being DC-DC converters, is designed to achieve this goal. A conceptual example of the power conversion tree for a smartphone is depicted in Figure 2.

Typical DC-DC converters in modern smartphones can be classified into two types, switching-mode DC-DC converters (simply called DC-DC converter) and low-dropout linear regulators (LDO), according to the circuit implementation and operation principles. A DC-DC converter consists of an inductor, capacitors, two MOSFET switches and a pulse-width-modulation (PWM) controller. This type of converter can step-up the output voltage so that it becomes higher than the input voltage (i.e., boost), or step-down the output voltage so that it is lower than the input voltage (i.e., buck). On the other hand, the output voltage of an LDO can only be lower than its input voltage. In general, the LDO has lower power conversion efficiency. Nevertheless, the LDO is an indispensable component in smartphones in that it can provide low-noise output voltage, and therefore, the LDO is the most suitable type of converter to provide power for some noise-sensitive RF or analog modules.

\subsection{DC-DC converter power loss model}

The power loss model of a PWM DC-DC converter is wellstudied in [8]. In general, the major sources of power loss in a DC-DC converter are conduction loss, switching loss in the power switches, and controller power loss, denoted by $P_{\text {conduction }}, P_{\text {switching }}$, and $P_{\text {controller }}$, respectively. The power loss of the buck converter,

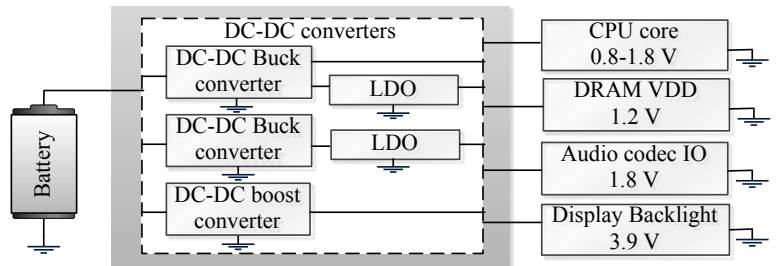

Figure 2: Conceptual diagram of a smartphone power conversion tree.

$P_{\text {buck }}$, can be expressed as:

$$
\begin{aligned}
P_{\text {buck }}= & P_{\text {conduction }}+P_{\text {switching }}+P_{\text {controller }} \\
= & I_{\text {out }}{ }^{2}\left(R_{L}+D R_{s w 1}+(1-D) R_{\text {sw } 2}\right) \\
& +(\Delta I)^{2}\left(R_{L}+D R_{s w 1}+(1-D) R_{s w 2}+R_{C}\right) / 12 \\
& +V_{\text {in }} f_{S}\left(Q_{s w 1}+Q_{s w 2}\right)+V_{\text {in }} I_{\text {controller }},
\end{aligned}
$$

where $D=V_{\text {out }} / V_{\text {in }}$ is the PWM duty ratio of the power switch, and $V_{\text {in }}$ and $V_{\text {out }}$ denote the input and output voltages, respectively; $I_{\text {out }}$ is the output current through the inductor; $\Delta I=(1-D) V_{\text {out }} /\left(L_{f} f_{s}\right)$ is the amplitude of the maximum current ripple at the inductor; $f_{s}$ is the switching frequency; and $I_{\text {controller }}$ denotes the current used in the control logic section of the converter. Series resistances of the inductor $L$ and capacitor $C$ are denoted by $R_{L}$ and $R_{C}$, respectively. Similarly, series resistances of the two MOSFET switches are represented by $R_{s w 1}$ and $R_{s w 2}$, respectively, while the amounts of their gate charge are denoted by $Q_{s w 1}$ and $Q_{s w 2}$, respectively. The first and second terms of (2) are DC and AC conduction losses, respectively; third term of (2) denotes the switching loss; while the last term of (2) corresponds to the controller power loss.

The power loss of the boost converter, $P_{\text {boost }}$, is modeled similarly except for the switching duty calculation, which is given by:

$$
\begin{aligned}
P_{\text {boost }}= & \left(\frac{I_{\text {out }}}{1-D}\right)^{2}\left(R_{L}+D R_{s w 1}+(1-D) R_{s w 2}+D(1-D) R_{C}\right) \\
& +\frac{(\Delta I)^{2}}{12}\left(R_{L}+D R_{s w 1}+(1-D) R_{s w 2}+(1-D) R_{C}\right) \\
& +V_{\text {in }} f_{s}\left(Q_{s w 1}+Q_{s w 2}\right)+V_{\text {in }} I_{\text {controller }},
\end{aligned}
$$

where $D=1-V_{\text {in }} / V_{\text {out }}$ and $\Delta I=\left(V_{\text {in }} D\right) /\left(L_{f} f_{s}\right)$.

As a result, the efficiency of a DC-DC converter, $\eta_{\text {switching }}$, can be calculated as:

$$
\eta_{\text {switching }}=\frac{V_{\text {out }} I_{\text {out }}}{V_{\text {in }} I_{\text {in }}}=\frac{V_{\text {in }} I_{\text {in }}-P_{\text {converter }}}{V_{\text {in }} I_{\text {in }}},
$$

where $I_{\text {in }}$ is the input current, and $P_{\text {converter }}$ is either $P_{\text {buck }}$ or $P_{\text {boost }}$ depending on the type of DC-DC converter used.

\subsection{LDO power loss model}

A typical LDO consists of an error amplifier, a pass transistor, and a feedback resistor network. The power loss of the LDO, denoted by $P_{l d o}$, is given by:

$$
P_{l d o}=I_{\text {out }}\left(V_{\text {in }}-V_{r e f} k\right)+I_{q} V_{\text {in }},
$$

where $V_{\text {ref }}$ is the reference voltage in the error amplifier; $k=\left(R_{1}+\right.$ $\left.R_{2}\right) / R_{2}$ corresponds to the voltage divider's gain coefficient, and $I_{q}$ denotes the quiescent current of the LDO. Unlike the switching converter in which the MOSFET switches dominate the total power loss, the pass transistor in the LDO has negligible impact on its total power loss [8]. Therefore, the power loss due to internal resistance of the pass transistor is not accounted for in the model. Thus the conversion efficiency of the LDO, $\eta_{l d o}$, may be expressed as:

$$
\eta_{\text {ldo }}=\frac{V_{\text {out }} I_{\text {out }}}{V_{\text {in }} I_{\text {in }}}=\frac{k V_{\text {ref }} I_{\text {out }}}{V_{\text {in }}\left(I_{\text {out }}+I_{q}\right)}
$$




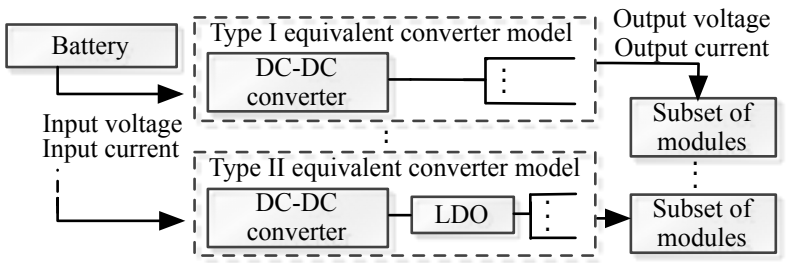

Figure 3: Types I and II equivalent power converter models.

\section{CHARACTERIZATION OF THE POWER CONVERSION EFFICIENCY}

Power converters including switching-mode DC-DC converters exhibit different conversion efficiency as a function of the load current [8]. A module is often powered through a set of converters from a battery source as shown in Figure 3. The power converter set can be an empty set (direct connection), single DC-DC converter, (more commonly) a cascade (series) connection of a DCDC converter and an LDO, (rarely) a cascade connection of various DC-DC converters, etc. Characterization of each power converter efficiency is not a trivial work unless the power conversion tree structure and converter specifications, and all the node voltages and branch currents of the conversion tree are available. Such a white-box approach is generally not possible for commercial smartphones. Although the Qualcomm MDP provides measurement of the device current values, the power conversion tree structure is not available for examination/measurements.

In this paper, we attempt a gray-box approach introducing an equivalent power converter concept. The equivalent converter models a set of power converters from the battery source to each module. In other words, the proposed equivalent power converter abstraction treats the set of power converters as a single equivalent converter. The abstraction enables a gray-box approach by which one can group modules in a (smartphone) system by their required supply voltage levels, which can be obtained from datasheets. Power conversion efficiency improvement in the subsequent optimization procedure can be effectively performed once we identify the power conversion efficiency of all the power conversion paths from the battery source to various modules in the system.

\subsection{Equivalent converter model}

We classify the equivalent converter models either a single DCDC converter, or a cascaded DC-DC converter and an LDO, named Type I and Type II equivalent converters, respectively. We assume that the battery output current goes through a voltage regulator in order to produce a constant voltage throughout its full discharge cycle. Without loss of generality, Types I and II equivalent converter models can represent most power conversion tree structures $[9,10$, 11]. Most digital logic components can be powered by a single DC-DC converter from the battery to the module - this gives rise to Type I converter model. A cascade of two or more DC-DC converters are rare, because increasing the number of cascade DC-DC converters increases the cost and form factor overhead with little (or no) benefit in terms of conversion efficiency. LDOs are often an indispensable component to provide low-ripple output voltage for switching noise-sensitive RF and analog modules. It is uncommon to use a single LDO from the battery to a device due to large dropout voltage. Instead, the conversion of the battery voltage to an initially higher target voltage using a DC-DC converter and subsequent use of LDO for the final power conditioning is a more efficient way of doing this - hence, our emphasis on Type II converter model.

According to (2), (3), and (5), the power loss of the equivalent converter can be expressed as:

$$
P_{e q v}=A\left(\alpha I_{q}+\sum_{i=1}^{N} I_{m o d, i}\right)^{2}+\alpha \beta \sum_{i=1}^{N} I_{m o d, i}+\left(B+\alpha \gamma I_{q}\right),
$$

where $N$ is the number of modules connected to the equivalent converter; $I_{m o d, i}$ is the input current of the $i^{\text {th }}$ module; $A$ is determined
Table 1: Grouping results for Snapdragon MDP MSM8660.

\begin{tabular}{|c|c|c|}
\hline Group & Modules & Voltage \\
\hline 1 and 2 & Group1: CPU core0 and Group2: CPU core1 & $0.8-1.225 \mathrm{~V}$ \\
\hline 3 & $\begin{array}{c}\text { Internal Memory, Audio DSP, and } \\
\text { Digital core (includes GPU and modems) }\end{array}$ & $1.1 \mathrm{~V}$ \\
\hline 4 & $\begin{array}{c}\text { Audio codec Vdd, LPDDR2 Vdd, ISM Vdd, } \\
\text { DRAM Vdd2, and Camera digital }\end{array}$ & $1.2 \mathrm{~V}$ \\
\hline 5 & $\begin{array}{c}\text { Audio codec IO, IO PAD3, } \\
\text { Display IO, DRAM Vdd1, } \\
\text { Camera IO, PLL, and eMMC host interface }\end{array}$ & $1.8 \mathrm{~V}$ \\
\hline 6 & $\begin{array}{c}\text { Audio codec analog, Haptic, SD card, } \\
\text { Touch screen, eMMC (Flash), IO PAD2, } \\
\text { SD card, and Ambient light sensor }\end{array}$ & $2.85 \mathrm{~V}$ \\
\hline 7 & Display Memory and Display backlight & $3.8 \mathrm{~V}$ \\
\hline
\end{tabular}

by the type of the DC-DC converter such that $A=R_{L}+D R_{s w 1}+$ $(1-D) R_{s w 2}$ for buck converter and $A=(1 /(1-D))^{2}\left(R_{L}+D R_{s w 1}+\right.$ $\left.(1-D) R_{s w 2}+D(1-D) R_{C}\right)$ for boost converter; $B$ is the sum of the second, third, and last terms of (2) or (3); $\alpha=0$ for Type I, and $\alpha=1$ for Type II; $\gamma$ is the input voltages of the LDO; and $\beta=\left(\gamma-V_{r e f} k\right)$. We can further simplify (7) by defining the output current of the equivalent converter, $I_{\text {eqvout }}=\sum_{i=1}^{N} I_{m o d, i}$, and thus, the power loss for both types of equivalent converter models can be expressed as:

$$
P_{\text {eqv }}=a I_{\text {eqv_out }}{ }^{2}+b I_{\text {eqv } \_ \text {out }}+c,
$$

where the coefficients $a, b$, and $c$ are derived from (7), and are largely dependent on the power converter design specification such as the power MOSFET gate width, inductor IR loss, controller loss, etc. [8]. Calculating those coefficients is the key step in of the power conversion efficiency characterization.

\subsection{Power converter grouping and regression analysis}

Measurement (or estimation) of the output current of all the equivalent power converters enables us to calculate the unknown coefficients of the equivalent power converter model. Once again, the input and output voltage levels of each equivalent power converters can be obtained from the device datasheets. The Qualcomm Snapdragon MDP MSM8660 [12] incorporates embedded power sensors that monitor and report current values of different modules with fine granularity. When the target smartphone does not provide embedded current sensors, we can estimate the module current values by activity profiling $[1,2,3,4]$.

Profiling various applications, which result in diverse usage patterns of the system modules, provides sufficient information and data to perform regression analysis and obtain the unknown coefficients. Linear regression analysis is a widely used method in system identification, requiring (i) a well-designed model and (ii) sufficient experimental data to extract the best-fit model coefficients. In reality, however, independent control of each module is a challenging task due to the lack of direct control knobs. For example, if we run an application that activates a camera module, the CPU, GPU, memory, and other associated component currents also ramp up and down. We must thus apply linear regression analysis to the whole system (including all smartphone modules) simultaneously, while trying to vary the activity level of each module by running different applications. However, this method may not produce sufficient data to cover the full range of activities for all smartphone modules, especially when the number of modules is large (e.g., the Snapdragon MDP MSM8660 has 27 embedded modules.) This is a potential source of inaccuracy for regression analysis due to the weak training set issue.

We tackle the problem by doing a module grouping in order to reduce the number of unknown coefficients that must be determined during the characterization process. This grouping procedure reduces the burden in terms of generating sufficient data for performing the linear regression analysis. The idea is that system modules that require the same operating voltage level can be combined into one group, and each group of modules is connected to the battery source via a single equivalent converter, as illustrated in Figure 3. 
This method matches well with low power design practices that try to minimize the number of power converters, due to their cost and internal power losses.

Given that the number of different voltage levels required by various modules in a smartphone platform is typically less than 10 [9, $11]$, the grouping method significantly reduces the number of parameters to be determined in linear regression. For example, the Snapdragon MDP MSM8660 requires only seven groups although the module count is 27 .

Finally, the total power loss of the smartphone, $P_{\text {loss }}$, is given by,

$$
P_{\text {loss }}=\sum_{k=1}^{G} P_{\text {eqv }, k}=\sum_{k=1}^{G}\left(a_{k} I_{\text {eqv_out }, k}{ }^{2}+b_{k} I_{\text {eqv_out }, k}+c_{k}\right),
$$

where $G$ is the number of groups; $P_{e q v, k}$ is the power loss of the $k^{\text {th }}$ equivalent converter corresponding to the $k^{\text {th }}$ group of modules; $I_{\text {eqvoout }, k}$ denote the output current of the equivalent converter, which can be measured using embedded sensors in the Snapdragon MDP MSM8660; $a_{k}, b_{k}$, and $c_{k}$ are the coefficients of the equivalent converter model (to be determined by linear regression.) We treat the battery voltage presented to the power conversion tree as being (nearly) constant, which is valid considering the function of the regulator between the battery cell/pack and the equivalent converter, therefore, we may assume that $a_{k}, b_{k}$, and $c_{k}$ are constant values.

\subsection{Experimental results}

\subsubsection{Experimental setup}

We use the Snapdragon MDP MSM8660 (in short, MDP) as a target platform. It is a cutting-edge smartphone platform equipped with Google Android OS 2.3 on top of Snapdragon 1.5 GHz asynchronous dual-core CPU, a 3D-supporting GPU, 3.61" WVGA multi-touch screen, $1 \mathrm{~GB}$ internal RAM, $16 \mathrm{~GB}$ on-board flash, WiFi, Bluetooth, a GPS, dual-side cameras, etc. We perform power measurement of each module using the application profiling tool named Trepn $^{\mathrm{TM}}$. Use of Trepn ${ }^{\mathrm{TM}}$ ensures higher accuracy of the measurements. Note, however, that our proposed method is independent of the measurement tools, e.g., we may use activity profiling for power measurement provided by Google or based on techniques presented in the literature $[1,2,3,4]$.

\subsubsection{Coefficient identification}

As shown in Table 1, the MDP modules may be classified into seven groups based on their operating voltage levels. Some modules such as the CPU cores in the MDP use dynamic voltage and frequency scaling (DVFS) techniques that require a range of variable supply voltage levels. Consequently, we keep each CPU core in a separate group but treat the equivalent converters of these groups identical to each other. Group 7 is associated with display, and therefore, the backlight luminance level mostly determines the current demand in this group. Group 7 coefficients are easy to identify because we can independently control the brightness of the display. In other words, we first perform the linear regression to identify coefficients of the equivalent converter model of Group 7, separately from the other groups.

For the remaining six groups, we profile various applications and collect sufficient data for the regression analysis as explained earlier. It is difficult to identify every $c_{k}$ coefficient of the $k^{\text {th }}$ equivalent converters directly from the linear regression process. Rather, we only extract $c_{\text {ext }}$ that corresponds to the sum of all the constant terms in (9), i.e., $c_{e x t}=\sum_{k=1}^{G} c_{k}$. We find an approximate value for each $c_{k}$ as $c_{k}=c_{\text {ext }}\left(P_{\text {group }, k} / P_{\text {group }, \text { total }}\right)$, where $P_{\text {group }, k}$ denotes the power consumption of Group $k$, and $P_{\text {group }, \text { total }}$ is the total power consumption of all the groups. The $P_{\text {group }, k}$ and $P_{\text {group }, \text { total }}$ values are available from the embedded sensors in the MDP.

The extracted coefficients of the seven equivalent converters are reported in Table 2. The power conversion efficiency of Group $k$, derived from $\left(P_{\text {group }, k} /\left(P_{\text {group }, k}+P_{\text {eqv }, k}\right)\right)$, is shown in Figure 4. We verify the characterization results of each equivalent power

Table 2: Extracted coefficients for each group.

\begin{tabular}{|c|c|c|c||c|c|c|c|}
\hline $\mathrm{k}$ & $a_{k}$ & $b_{k}$ & $c_{k}$ & $\mathrm{k}$ & $a_{k}$ & $b_{k}$ & $c_{k}$ \\
\hline 1,2 & 0.4427 & 0.0025 & 0.0170 & 5 & 0.1971 & 0.5232 & 0.0128 \\
\hline 3 & 0.4079 & 0.1742 & 0.0675 & 6 & 0.1814 & 0.2928 & 0.0320 \\
\hline 4 & 0.1152 & 0.1757 & 0.0077 & 7 & 0.4091 & 0.3871 & 0.0289 \\
\hline
\end{tabular}

(a) Groups 1 and 2

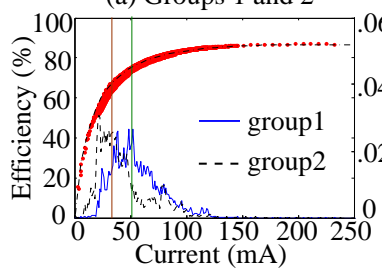

(c) Group 4

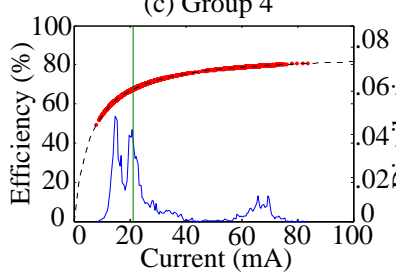

(e) Group 6

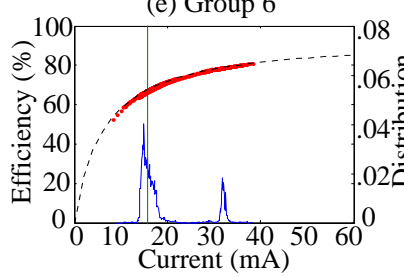

Model, • Measured efficiency, —-Measured current distribution,- - Mean

Figure 4: Conversion efficiencies for all groups.

converters. Figure 5 shows the comparison of the system power consumption trace between the real measurement as reported by a built-in battery sensor and the estimation as obtained by our extracted equivalent converter coefficients. We have thus confirmed that the results of the power conversion efficiency characterization process is accurate enough for the subsequent optimization process.

\section{POWER CONVERTER TUNING}

Power converter tuning reduces power loss without any performance degradation. This is because, unlike typical low-power design techniques that often exploit a tradeoff between performance/service quality and power efficiency, the power converter tuning does not utilize slack time of the system.

Enhancement of the overall efficiency of a DC-DC converter can greatly increase the overall system power efficiency $[13,9]$. DCDC converters show very high overall efficiency under the right operating conditions. However, their efficiency can be low if they are operated outside the recommended range on input and output voltages and load currents [8]. Therefore, ensuring that each DC-DC converter in the system is operating under the right operating conditions is an effective way of improving the system power efficiency. For example, Reference [10] presents a dynamic programing ap-

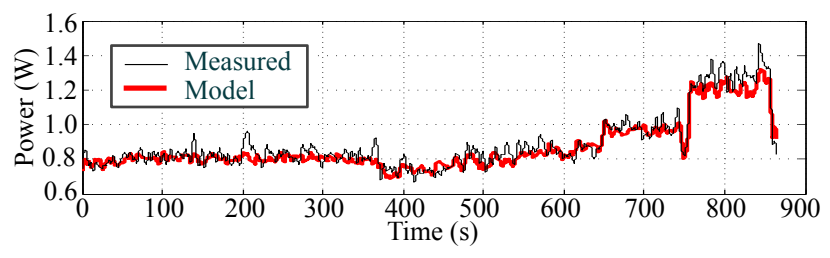

Figure 5: A part of traces of total power consumption: measured data and modeled data. 


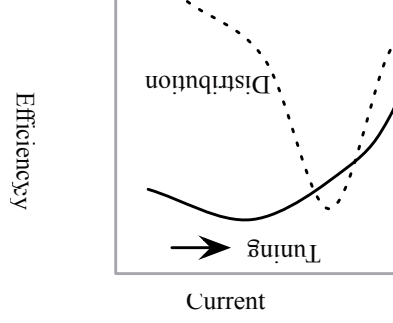

(a) $W$ is over designed.

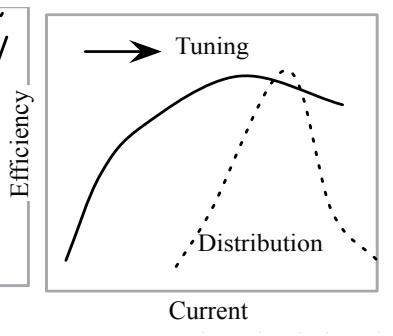

(b) $W$ is under designed.
Figure 6: Concept of the width tuning.

proach to design the structure of the power conversion tree in a system while at the same time selecting the 'optimal' DC-DC converter or LDO for each node of the conversion tree. Reference [14] proposes the concept of parallel connections of high frequency DCDC converters for distributed energy storage systems. In contrast, the present paper starts with a fixed conversion tree structure, but uses load currents demands and converter characteristics to perform MOSFET switch sizing so as to improve the overall efficiency of the power conversion process in a smartphone system.

As stated above, we focus on the optimal assignment of the gate width, $W$, of the MOSFET switches in a DC-DC converter. The pass transistor in the LDO has negligible impact on its power loss, therefore, we do not take it into account. (c.f. Section 2.2.) As we previously depicted in Figure 4, DC-DC converters in the MDP are not properly tuned in regard to the actual Android applications running on it. The width tuning, which was introduced in [8], is conceptually explained in Figure 6. The idea is to match the value of $W$ in the equivalent converter such that the desirable operating conditions of the equivalent converter match with the current distribution produced by the actual usage profiles of various Android applications in order to yield the maximum conversion efficiency for the typical daily use of a smartphone.

Sizing down $W$ causes the turn-on resistance of the MOSFET increased and the gate charge decreased. (i.e., $W=\left(W_{o} R_{o}\right) / R_{S w 1,2}=$ $\left(W_{o} Q_{s w 1,2}\right) / Q_{o} ; R_{o}$ and $Q_{o}$ correspond to the turn-on resistance and the gate charge of a MOSFET with a gate size of $W_{o}$, respectively.) As a function of $W$, we can express the DC-DC converter power loss models, (2) and (3), as:

$$
P_{\text {converter }}=\left(\frac{r_{1}}{W}+r_{2}\right) I_{\text {out }}^{2}+r_{3} W+r_{4},
$$

where $I_{\text {out }}$ denotes the output current of the DC-DC converter; $r_{1}$, $r_{2}, r_{3}$, and $r_{4}$ are constants.

Given that the two MOSFET switches in a DC-DC converter dominate the power loss of the equivalent converter, and $I_{q}$ is small, we rewrite (8) as:

$$
P_{\text {eqv }, k}=\left(\frac{r_{1, k}}{W_{k}}+r_{2, k}\right) I_{\text {eqvout }, k}{ }^{2}+b I_{\text {eqvout }, k}+r_{3, k} W_{k}+r_{4, k},
$$

where $P_{e q v, k}$ and $I_{e q v \_o u t, k}$ are the power loss and the output current of the $k^{\text {th }}$ equivalent converter, respectively, corresponding to the $k^{t h}$ group of modules; $W_{k}, r_{1, k}, r_{2, k}, r_{3, k}$ and $r_{4, k}$ are the coefficients of the equivalent converter model that have been determined

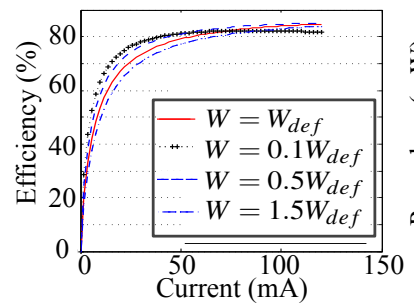

(a) Efficiency : Group 7

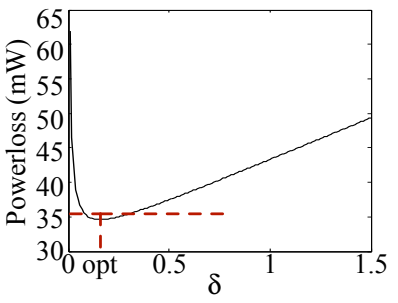

(b) Power loss : Group 7
Figure 7: Relation between the power conversion efficiency and W: Group 7.
Table 3: $W_{\text {def }}$ of the equivalent converter models.

\begin{tabular}{|c|c|c|c|c|c|c|}
\hline Group & 1 and 2 & 3 & 4 & 5 & 6 & 7 \\
\hline$W_{\text {def }}$ & 1.2401 & 1.1033 & 1.3109 & 1.4033 & 1.4102 & 0.7368 \\
\hline
\end{tabular}

based on linear regression. The resultant coefficient $W_{k}$ is the default value of $W\left(W_{d e f}\right)$ of the $k^{t h}$ equivalent converter, which is shown in Table 3.

\subsection{Width tuning}

Figure 7 (a) shows an example in which $W$ changes the efficiency graph of Group 7. Note that the efficiency of the DC-DC converter increases as $W$ becomes smaller (larger) in the relatively low (high) current demand region. Other groups show similar behaviors. Figure 7 (b) shows the relation between the power loss and $W$ under the condition that the output current of Group 7 is $37 \mathrm{~mA}$. Here, $\delta$ is a control variable such that the modified value of $W$ is calculated as $\delta \cdot W_{d e f}$. The power loss plots have a convex functional form in terms of $W$, which implies that there is a globally optimal value of $W, W_{\text {opt }}$, that minimizes the power loss of the equivalent converter. Given that $I_{\text {eqvout }}$ is a random variable, our goal of finding $W_{\text {opt }}$ for the actual usages of the various applications should be based on the probability distribution of $I_{\text {eqv out }}$ acquired by the usages of the applications. Such probability distribution can be obtained via real measurements, yielding the current profile of each module (thereby, each group of modules) when each type of application is running. We run 10 representative smartphone applications and collect the probability distribution of $I_{\text {eqv_out }}$ for each group of modules and for each application.

Throughout the module current profiles collection and (11), the expected power loss of an equivalent converter can be generally expressed as:

$$
E\left[P_{\text {eqv }}\right]=\left(\frac{r_{1}}{W}+r_{2}\right) E\left[I_{\text {eqv_out }}{ }^{2}\right]+b E\left[I_{\text {eqv_out }}\right]+r_{3} W+r_{4} .
$$

where $E\left[I_{\text {eqvoout }}\right]$ denotes the expected value of $I_{\text {eqvoout }}$, and $E\left[I_{\text {eqv_out }}{ }^{2}\right]$ is the expected value of $I_{\text {eqv_out }}^{2}$.

To find $W_{\text {opt }}$, we limit $\delta$ to two decimal places to avoid high computation cost (also in practice, two decimal place precision is quite adequate.) We define the power conversion efficiency enhancement by Gain $_{\eta}=100\left(\eta_{\text {optimal }} / \eta_{\text {original }}-1\right)$ and power loss reduction by Gain $_{P}=100\left(1-P_{\text {optimal }} / P_{\text {original }}\right)$, where the optimal power conversion efficiency, $\eta_{\text {optimal }}$, and power loss, $P_{\text {optimal }}$, are derived from (10) with the optimal value of $\delta$, and the original power conversion efficiency, $\eta_{\text {original }}$, and power loss, $P_{\text {original }}$ are derived from (10) with $\delta=1$.

\subsection{DC-DC converter tuning results}

Table 4 shows an example of the width tuning that each $W_{\text {opt }}$ is derived from each group and four different applications, 'Clock', 'Call', 'Facebook', and 'Skype-videochat'. As each application causes different current distribution, the values of $W_{\text {opt }}$ for the same group are different. Table 4 also shows the tuning results for the four applications. According to the resulted $W_{\text {opt }}$ for each group, the Gain $_{\eta}$ and Gain $_{P}$ columns show the resultant gains for each group. The upper bound of the power conversion efficiency enhancement $\left(\right.$ Gain $\left._{\eta, \max }\right)$ and the power loss reduction $\left(\right.$ Gain $\left._{p, \max }\right)$ are obtained from the distribution of $I_{\text {eqvout }}$ and $W_{\text {opt }}$.

We collect the current distribution data from 10 applications, including the four applications named in Table 4, 'Camera', 'GoogleMap', 'Neocore', 'SMS', 'System setting', and 'Youtube'. All the applications except 'Clock' and 'System setting' are run under the same setup where WiFi is turned on and the backlight level of the display is the highest. 'Clock' is measured under the median level of the backlight and WiFi on, whereas 'System setting' is measured under the lowest backlight and WiFi off. For the case of 'Call', we consider auto turn-off screen during the call. We, then, classify the 10 applications into seven categories, i) communication (contains 'SMS', 'Call', and 'Skype-videochat'), ii) browsing (contains 'Web browsing'), iii) media (contains 'Camera' and 'Youtube'), 
Table 4: Example of DC-DC converter tuning results of four types of applications.

\begin{tabular}{|c|c|c|c|c|c|c|c|c|c|c|}
\hline & \multicolumn{5}{|c|}{ Clock } & \multicolumn{5}{|c|}{ Call } \\
\hline Group & $W_{o p t}$ & $\operatorname{Gain}_{\eta}$ & $\operatorname{Gain}_{P}$ & Gain $_{\eta, \max }$ & $\operatorname{Gain}_{P, \max }$ & $W_{o p t}$ & Gain $_{\eta}$ & $\operatorname{Gain}_{P}$ & $\operatorname{Gain}_{\eta, \max }$ & $\operatorname{Gain}_{P, \max }$ \\
\hline 1 & 0.2356 & 11.3508 & 34.5181 & 27.2733 & 43.1362 & 0.2852 & 8.7073 & 30.6121 & 23.7710 & 40.8894 \\
\hline 2 & 0.1369 & 21.1175 & 42.8718 & 31.2166 & 47.0092 & 0.1991 & 12.9707 & 37.3828 & 27.1296 & 44.5621 \\
\hline 3 & 0.3751 & 6.7329 & 15.7217 & 13.5717 & 24.4609 & 0.3751 & 6.6543 & 15.5894 & 13.5717 & 24.4609 \\
\hline 4 & 0.1180 & 15.2996 & 34.9625 & 19.4536 & 38.4971 & 0.1704 & 10.2327 & 28.7502 & 12.4670 & 31.7882 \\
\hline 5 & 0.1123 & 15.5010 & 30.7496 & 17.9247 & 33.0915 & 0.1263 & 13.0017 & 27.9058 & 15.4971 & 30.7201 \\
\hline 6 & 0.1128 & 14.6603 & 34.4909 & 15.9501 & 35.8211 & 0.1269 & 14.0235 & 33.7792 & 16.0955 & 35.9118 \\
\hline 7 & 0.0884 & 6.9630 & 23.5545 & 8.1285 & 25.3117 & - & - & - & - & - \\
\hline Overall & - & 9.9381 & 24.8974 & 15.0994 & 30.8775 & - & 9.3516 & 23.5594 & 16.2542 & 30.9136 \\
\hline Group & \multicolumn{5}{|c|}{ Facebook } & \multicolumn{5}{|c|}{ Skype-videochat } \\
\hline 1 & 0.4216 & 4.4201 & 20.7700 & 11.9557 & 32.7724 & 0.4216 & 4.3408 & 20.544 & 12.8354 & 33.2795 \\
\hline 2 & 0.2862 & 8.4227 & 30.8524 & 14.4969 & 38.0183 & 0.4230 & 4.1481 & 20.9525 & 13.4634 & 33.9652 \\
\hline 3 & 0.3862 & 6.0869 & 14.5694 & 13.3955 & 24.1807 & 0.3751 & 6.6947 & 15.6603 & 13.5717 & 24.4609 \\
\hline 4 & 0.2097 & 8.5744 & 25.9577 & 14.0064 & 33.1636 & 0.5375 & 1.6203 & 7.4004 & 2.5731 & 10.9563 \\
\hline 5 & 0.1263 & 12.514 & 27.2880 & 15.7297 & 30.9577 & 0.1824 & 8.4489 & 21.3063 & 9.7984 & 23.4784 \\
\hline 6 & 0.1128 & 14.3447 & 34.1397 & 15.9733 & 35.8437 & 0.2256 & 6.0727 & 24.9642 & 6.4484 & 25.8884 \\
\hline 7 & 0.2210 & 1.9788 & 10.5936 & 2.0172 & 10.7499 & 0.1179 & 4.9756 & 19.7093 & 6.4110 & 22.5335 \\
\hline Overall & - & 5.7545 & 19.0159 & 8.8194 & 25.0937 & - & 5.3158 & 17.9998 & 9.0870 & 24.6192 \\
\hline
\end{tabular}

Table 5: DC-DC converter tuning results of Patterns I and II.

\begin{tabular}{|c|c|c|c|c|c|}
\hline Group & $W_{\text {opt }, I}$ & Gain $_{\eta}$ & Gain $_{P}$ & Gain $_{\eta, \text { max }}$ & Gain \\
\hline, max \\
\hline 1 & 0.2976 & 7.9717 & 29.7824 & 26.2208 & 40.8462 \\
\hline 2 & 0.1886 & 14.047 & 38.7954 & 43.2898 & 46.5719 \\
\hline 3 & 0.3751 & 6.4011 & 15.1687 & 13.8601 & 24.7137 \\
\hline 4 & 0.1704 & 10.5933 & 29.3017 & 22.2939 & 39.5304 \\
\hline 5 & 0.1263 & 12.8753 & 27.7511 & 17.7792 & 32.8968 \\
\hline 6 & 0.1269 & 13.9486 & 33.7017 & 19.3969 & 38.6310 \\
\hline 7 & 0.2137 & 2.0874 & 11.0332 & 6.8824 & 21.6813 \\
\hline Overall & - & 6.2176 & 20.0263 & 13.6376 & 29.4715 \\
\hline \hline Group & $W_{\text {opt,II }}$ & Gain & Gain & Gain & \\
\hline 1 & 0.3348 & 6.4977 & 26.7376 & 32.3645 & Gain \\
\hline 2 & 0.2115 & 6.1590 & 37.0320 & 33.1697 & 44.9638 \\
\hline 3 & 0.3862 & 9.4790 & 14.7303 & 13.6770 & 24.4264 \\
\hline 4 & 0.1835 & 12.4529 & 27.5864 & 22.0385 & 39.1593 \\
\hline 5 & 0.1263 & 13.6757 & 27.2171 & 17.7792 & 32.8968 \\
\hline 6 & 0.1269 & 2.0615 & 33.3899 & 19.3969 & 38.6310 \\
\hline 7 & 0.2137 & 2.0874 & 10.9291 & 6.8824 & 21.6813 \\
\hline Overall & - & 6.2955 & 20.0705 & 15.2870 & 30.0543 \\
\hline
\end{tabular}

iv) productivity (contains 'Clock'), v) system (contains 'System setting'), vi) games (contains 'Neocore'), and vii) maps (contains 'GoogleMap'). We determine their run time according to the two types of usage patterns collected by i) deploying a custom logger on the phones of 33 Android users (Pattern I), ii) 222 Windows Mobile users across different demographics (Pattern II) in [15].

The current distribution from Pattern I is shown in Figure 4. The original power conversion efficiencies of Patterns I and II are $70.7410 \%$ and $70.4817 \%$, respectively. Table 5 shows the optimization results for both Patterns I and II, where the values of $W_{o p t}$ are $W_{o p t, I}$ for Pattern I, and $W_{o p t, I I}$ for Pattern II. The overall power conversion efficiency enhancements are from $6 \%$ to $15 \%$, which correspond to from $20 \%$ to $30 \%$ power loss reduction during power conversion, respectively.

\section{CONCLUSIONS}

This paper demonstrated that significant power loss occurs during power conversion from the battery source to various devices in a modern smartphone. To mitigate this problem, the paper introduced a systematic method for system-level power conversion efficiency enhancement in smartphones. First, we proposed the equivalent power converter concept that abstracts the power conversion path from the battery to the modules into a single equivalent power converter. This enabled us to identify the model coefficients from application profiling. We demonstrated the accuracy of power conversion efficiency characterization and how the current power converter setup deviates from the optimal operating conditions. Finally, we proposed a power converter tuning procedure that resulted in $6 \%$ to $15 \%$ overall power conversion efficiency en- hancement, which translates to up to $30 \%$ power loss reduction during power conversion. As part of the future work, the optimization procedure will be improved by considering a multitude of different applications and usage scenarios.

\section{ACKNOWLEDGEMENTS}

This work is sponsored by a grant from the U.S. National Science Foundation, the Brain Korea 21 Project, the Smart IT Convergence System Research Center funded by the Ministry of Education, Science and Technology as Global Frontier Project (SIRC2011-0031851), and ICT at Seoul National University.

\section{REFERENCES} user activity patterns to guide power optimizations for mobile architectures," MICRO, 2009.

[2] L. Zhang and et al., "Accurate online power estimation and automatic battery behavior based power model generation for smartphones," CODES/ISSS, 2010.

[3] M. Dong and L. Zhong, "Self-constructive high-rate system energy modeling for battery-powered mobile systems," MobiSys, 2011.

[4] A. Pathak, Y. C. Hu, M. Zhang, P. Bahl, and Y. Wang, "Fine-grained power modeling for smartphones using system call tracing," EuroSys, 2011

[5] W. Yuan and K. Nahrstedt, "Energy-efficient soft real-time CPU scheduling for mobile multimedia systems," SOSP, 2003.

[6] D. Shin, Y. Kim, N. Chang, and M. Pedram, "Dynamic voltage scaling of oled displays," DAC, 2011.

[7] I. Choi, H. Shim, and N. Chang, "Low-power color TFT LCD display for hand-held embedded systems," ISLPED, 2002.

[8] Y. Choi, N. Chang, and T. Kim, "DC-DC converter-aware power management for low-power embedded systems," IEEE T. on Computer-Aided Design of Integrated Circuits and Systems, 2007.

[9] C. Shi, B. C. Walker, E. Zeisel, E. B. Hu, and G. H. McAllister, "A highly integrated power management IC for advanced mobile applications," CICC, 2006.

[10] B. Amelifard and M. Pedram, "Optimal design of the power-delivery network for multiple voltage-island system-on-chips," IEEE T. on Computer-Aided Design of Integrated Circuits and Systems, 2009.

[11] TexasInstruments, "Handset:smartphone," http://www.ti.com/solution /handset_smartphone.

[12] Qualcomm, "Snapdragon MDP MSM8660 datasheet," https://devel oper.qualcomm.com/sites/default/files/snapdragon-mdp-8660.pdf.

[13] J. Xiao, A. Peterchev, J. Zhang, and S. Sanders, "An ultra-low-power digitally-controlled buck converter IC for cellular phone applications," APEC, 2004.

[14] Y. Du, M. Wang, R. T. Meitl, S. Lukic, and A. Q. Huang, "High-frequency high-efficiency dc-dc converter for distributed energy storage modularization," IECON, 2010.

[15] F. Hossein, M. Ratul, K. Srikanth, L. Dimitrios, G. Ramesh, and E. Deborah, "Diversity in smartphone usage," MobiSys, 2010. 